\title{
Distribuição Volumétrica de Pontas de Pulverização Turbo TeEJet 11002 em Diferentes Condições OPERACIONAIS ${ }^{1}$
}

\author{
Distribution Pattern of Nozzle TT 11002 under Different Operational Conditions
}

\author{
FREITAS, F.C.L. ${ }^{2}$, TEIXEIRA, M.M. ${ }^{3}$, FERREIRA, L.R. ${ }^{4}$, FERREIRA, F.A. ${ }^{4}$, MACHADO, A.F.L. ${ }^{5}$ e \\ VIANA, R.G. ${ }^{5}$
}

\begin{abstract}
RESUMO - Este trabalho teve como objetivo determinar a uniformidade de distribuição para a ponta de pulverização TT 11002 em função de espaçamento entre bicos, altura de barra e pressão de trabalho, bem como avaliar a possibilidade de redução do volume de calda por meio da alteração do espaçamento entre bicos. Foram avaliados os perfis de distribuição de pontas TT 11002, para as alturas de 30, 40 e $50 \mathrm{~cm}$, nas pressões de 100, 200, 300 e $400 \mathrm{kPa}$. A determinação foi realizada em mesa de teste para pontas de pulverização hidráulica, composta por canaletas em "V", espaçadas de $5 \mathrm{~cm}$. Foram utilizadas, para cada configuração, 10 unidades de pontas TT 11002, instaladas isoladamente no centro da mesa. Em seguida, procedeu-se à coleta e à medição do volume pulverizado por 60 segundos e determinou-se o perfil de distribuição. A partir do perfil de distribuição em cada uma das configurações estudadas, simulou-se, utilizando programa computacional, a deposição ao longo da barra de pulverização para bicos espaçados de 40, 50, 80, 100, 120 e $150 \mathrm{~cm}$. A uniformidade de distribuição foi avaliada pelo coeficiente de variação (CV), em uma barra de 12 metros de largura, onde foram utilizados os dados dos seis metros centrais. A ponta de pulverização TT 11002 apresentou boa uniformidade de distribuição (CV inferior a 7\%), na pressão de $100 \mathrm{kPa}$, para bicos espaçados até $50 \mathrm{~cm}$ e nas alturas de barra de 40 e $50 \mathrm{~cm}$. Nas pressões acima de $200 \mathrm{kPa}$, boa uniformidade de distribuição foi verificada para espaçamentos de até 50,100 e $120 \mathrm{~cm}$, para as alturas de barra de 30,40 e $50 \mathrm{~cm}$, respectivamente. A ponta TT 11002 possibilita redução de volume de calda com o aumento do espaçamento entre bicos, mantendo boa uniformidade de distribuição com maior capacidade operacional do equipamento de aplicação.
\end{abstract}

Palavras-chave: uniformidade de distribuição, tecnologia de aplicação, volume de aplicação, espaçamento entre bicos, coeficiente de variação.

\begin{abstract}
This work aimed to evaluate spray distribution uniformity for nozzle TT 11002 using varying nozzle spacing, spray height and pressure, as well as the possibility of reducing spray volume by increasing nozzle spacing. Nozzle deposition pattern was evaluated for 30,40 and $50 \mathrm{~cm}$ spray heights, at pressures of 100,200, 300 and $400 \mathrm{kPa}$, on a pattern table, using a "V" channel and $5 \mathrm{~cm}$ spacing. Ten nozzles were individually placed at the center of the pattern table followed by collection and measurement of the volume sprayed for 60 seconds and determination of the distribution pattern. Based on the deposition patterns, distribution uniformity for nozzles spaced at 40, 50, 80, 100, 120 and $150 \mathrm{~cm}$ was simulated using a computer program. Nozzle TT 11002 showed good distribution uniformity, with coefficient of variation (CV) below 7 at $100 \mathrm{kPa}$ pressure for nozzles spaced up to $50 \mathrm{~cm}$ and at 40 and $50 \mathrm{~cm}$ spray heights. At pressures above $200 \mathrm{kPa}$, good distribution uniformity was verified at up to 50,100 and $120 \mathrm{~cm}$ spacing at 30,40 and $50 \mathrm{~cm}$ spray heights, respectively. Nozzle TT 11002 allows spray volume reduction by increasing nozzle spacing, with good distribution uniformity and increased operational capacity of the spraying equipment.
\end{abstract}

Key words: distribution uniformity, application technology, application volume, nozzle spacing, coefficient of variation.

Recebido para publicação em 28.10.2004 e na forma revisada em 21.4.2005.

2 Pós-Graduando, D.S., do Dep. de Fitotecnia da Universidade Federal de Viçosa - UFV, bolsista do CNPq-Brasil, 36570-000 Viçosa-MG; ${ }^{3}$ Prof. do Dep. de Engenharia Agrícola da UFV; ${ }^{4}$ Prof. do Dep. de Fitotecnia da UFV; ${ }^{5}$ Pós-Graduando, M.S., do Dep. de Fitotecnia da UFV.

Planta Daninha, Viçosa-MG, v. 23, n. 1, p. 161-167, 2005 


\section{INTRODUÇÃO}

A aplicação correta de produtos fitossanitários pode melhorar sua eficácia biológica e reduzir danos causados às culturas vizinhas, ao meio ambiente e ao homem. Essa aplicação é realizada, normalmente, utilizando pulverização hidráulica, que é definida como "processo mecânico de geração de gotas" (Cordeiro, 2001; Matuo et al., 2001). As gotas são produzidas pelas pontas de pulverização, que também determinam a vazão e a distribuição do líquido pulverizado, sendo, portanto, o equipamento mais importante do pulverizador (Bauer \& Raetano, 2004).

A uniformidade de distribuição da calda, ao longo da barra de pulverização, é dada pelas condições de montagem e operação do equipamento, como espaçamento entre bicos, altura da barra, ângulo de abertura dos bicos e pressão de trabalho (Perecin et al., 1994; Bauer \& Raetano, 2004), sendo avaliada pelo coeficiente de variação da resultante da sobreposição de distribuição do conjunto de bicos colocados na barra. O padrão de uniformidade de distribuição utilizado em países da comunidade européia estabelece que o coeficiente de variação seja menor que $7 \%$. Distribuição desuniforme, abaixo do volume mínimo exigido, produz controle insuficiente, e quantidades acima causam perdas financeiras, toxidez nas culturas e danos ao meio ambiente (Cordeiro, 2001).

Atualmente, existe uma tendência à redução do volume de calda aplicado, que resulta em menor transporte de água ao campo e redução das paradas para reabastecimento do pulverizador, obtendo-se, com isso, diminuição do custo da aplicação e aumento da capacidade operacional do equipamento de aplicação (Matthews, 1979; Marochi, 1993; Lima \& Machado Neto, 2001), o que pode, em alguns casos, evitar a compra de tratores e pulverizadores (Marochi, 1993).

Diversos trabalhos têm sido desenvolvidos no sentido de avaliar efeitos de volumes de aplicação, em diferentes equipamentos de aplicação, no controle de plantas daninhas. Furlanetti et al. (2001), avaliando combinações de pontas de pulverização em barra de aplicação dirigida para o herbicida glyphosate, verificaram que arranjos que proporcionaram volumes de calda reduzidos, inferiores a $100 \mathrm{~L} \mathrm{ha}^{-1}$, destacaram-se como mais eficientes. Outros trabalhos com volumes de calda menores têm proporcionado maior eficácia do glyphosate no controle de plantas daninhas (Carlson \& Burnside, 1984; Mcwhorter \& Hanks, 1993).

Souza \& Dorneles (1995), avaliando os volumes de calda de 75,150 e $250 \mathrm{~L} \mathrm{ha}^{-1}$ na aplicação de misturas de bentazon com os herbicidas acifluorfen $\mathrm{Na}$, lactofen, fomesafen, imazethapyr, chlorimuron-ethyl, imazaquin e, também, da mistura chlorimuron-ethyl + acifluorfen $\mathrm{Na}$, aplicados em pós-emergência na cultura da soja, constataram que não houve efeito do volume de calda no controle do leiteiro (Euphorbia heterophylla) e de guanxuma (Sida sp.). No entanto, melhores respostas foram verificadas a 75 e $150 \mathrm{~L} \mathrm{ha}^{-1}$ para o controle do picão-preto (Bidens pilosa). Lima \& Machado Neto (2001) verificaram que o herbicida fluazifop-p-butil foi igualmente eficiente no controle de gramíneas anuais na cultura da soja quando aplicado nos volumes de calda de 100 e $200 \mathrm{~L} \mathrm{ha}^{-1}$.

Reduções no volume de calda podem ser alcançadas por meio do aumento da velocidade de deslocamento do pulverizador, da redução da pressão de trabalho e, principalmente, da utilização de pontas de baixa vazão, capazes de produzir gotas menores com boa cobertura do alvo. Deve-se, neste caso, ter mais atenção às condições ambientais, pois gotas menores estão mais sujeitas a perdas por deriva e por evaporação.

Outra estratégia para redução do volume de calda é o aumento do espaçamento entre bicos. Todavia, uma boa uniformidade de distribuição ao longo da barra somente é alcançada quando se é respeitada a inter-relação entre espaçamento de bicos, ângulo de abertura das pontas e altura da barra. Esta última, entre outros fatores, é dependente das condições ambientais, pois, aumentando-se a distância entre a ponta de pulverização e o alvo, maior será a influência delas sobre a deriva (Matuo et al., 2001). A angulação do jato produzido pelas pontas é um fator importante a ser considerado para o aumento do espaçamento entre bicos a uma mesma altura da barra de pulverização. 
As pontas de pulverização Turbo Teejet (TT) formam jato plano e são indicadas pelo fabricante (Spraying Sistems CO, 1999) para operar nas pressões de 100 a $600 \mathrm{kPa}$, produzindo gotas muito grossas a finas, respectivamente. São recomendadas para aplicação de herbicidas, inseticidas e fungicidas sistêmicos e herbicidas em pré-emergência, espaçadas de $0,50 \mathrm{~m}$, a $0,50 \mathrm{~m}$ de altura. Segundo Ferreira et al. (2003), as pontas TT podem trabalhar espaçadas de $1,0 \mathrm{~m}$, a $0,50 \mathrm{~m}$ do alvo para aplicação de herbicidas, aumentando com isso a capacidade operacional do pulverizador.

A maioria dos pulverizadores possui barras com bicos espaçados de 40 ou $50 \mathrm{~cm}$. Qualquer procedimento no sentido de alterar esse espaçamento é extremamente trabalhoso, podendo-se, neste caso, fazer a obstrução de bicos e trabalhar com espaçamentos múltiplos desses valores, como 80, 100 e $120 \mathrm{~cm}$, desde que se obtenha boa uniformidade de distribuição e cobertura do alvo suficiente para a eficácia do defensivo.

Este trabalho teve como objetivo determinar, em laboratório, o CV em função dos espaçamentos entre bicos, altura de barra e pressão de trabalho para a ponta TT 11002 , bem como avaliar a possibilidade de redução do volume de calda por meio da alteração do espaçamento entre bicos.

\section{MATERIAL E MÉTODOS}

O ensaio foi desenvolvido no Laboratório de Mecanização Agrícola da Universidade Federal de Viçosa, em Viçosa-MG, onde foram avaliados os perfis de distribuição volumétrica para a ponta TT 11002 , operando nas alturas de 30, 40 e $50 \mathrm{~cm}$, nas pressões de 100, 200, 300 e $400 \mathrm{kPa}$, num total de 12 configurações.

A determinação dos perfis de distribuição foi realizada em mesa de teste para pontas de pulverização hidráulica, composta por canaletas em "V", separadas entre si em cinco centímetros, construída de acordo com as Normas ISO 5.682-1:1996.

Foram utilizadas 10 unidades de pontas TT 11002, instaladas isoladamente no centro da mesa, de modo que o jato fosse lançado na posição vertical. Após a instalação de cada ponta, colocou-se o sistema hidráulico em funcionamento até que o fluxo do líquido se estabilizasse à pressão desejada, efetuandose então a coleta do volume pulverizado por 60 segundos, em provetas de $100 \mathrm{~mL}$ instaladas sob as canaletas coletoras, sendo a feita medição em provetas de $50 \mathrm{~mL}$.

Com base nos volumes médios coletados nas 10 repetições, em cada tratamento, foram determinados os perfis médios de distribuição volumétrica, possibilitando o cálculo do padrão médio de distribuição volumétrica ao longo da barra de pulverização, o qual foi determinado em programa computacional (Microsoft Excel), onde se fez a simulação da deposição na barra de pulverização, podendo-se trabalhar com um número variado de bicos, com espaçamentos variáveis de $5 \mathrm{~cm}$ (valor correspondente à largura da canaleta coletora). Neste trabalho, foram avaliadas as deposições ao longo da barra para bicos espaçados de 40, 50, 80, 100, 120 e $150 \mathrm{~cm}$, sendo os dois primeiros equivalentes aos espaçamentos geralmente encontrados nos pulverizadores, e os demais, obtidos a partir da obstrução de bicos na situação anterior.

A uniformidade de distribuição ao longo da barra foi avaliada pelo coeficiente de variação (CV) obtido por meio da fórmula: $\mathrm{CV}=($ desviopadrão/média)x100, em uma barra de $12 \mathrm{~m}$ de largura, com 30, 24, 15, 12, 10 e 8 bicos para os espaçamentos de 40, 50, 80, 100, 120 e $150 \mathrm{~cm}$ entre bicos, respectivamente, em que foram utilizados os dados dos seis metros centrais. Para os tratamentos que obtiveram CV inferior a $7 \%$, efetuou-se o cálculo do volume de aplicação obtido a partir das vazões médias para as pressões estudadas nas velocidades simuladas de 4 e $6 \mathrm{~km} \mathrm{~h}^{-1}$, visando verificar a possibilidade de redução do volume de aplicação através do aumento do espaçamento entre bicos.

\section{RESULTADOS E DISCUSSÃO}

Verifica-se, para todas as alturas da barra de pulverização, que, operando à pressão de $100 \mathrm{kPa}$, o padrão de deposição da ponta TT 11002 é contínuo, com uniformidade de volume no centro e quedas bruscas nas extremidades (Figura 1). Segundo Matuo et al. (2001), pontas com distribuição contínua são indicadas para aplicação em faixa, sem haver sobreposição com bicos vizinhos. Com isso,

Planta Daninha, Viçosa-MG, v. 23, n. 1, p. 161-167, 2005 
em aplicações dirigidas de produtos fitossanitários, que demandam menor cobertura do alvo, como os herbicidas aplicados em préemergência e produtos sistêmicos, essa ponta é um alternativa interessante, pois a $100 \mathrm{kPa}$ ela produz gotas grandes (Spraying Systems CO, 1989) e também permite operar a pequenas distâncias do alvo, devido ao seu grande ângulo de abertura, cobrindo uma faixa de 1,0 , 0,80 e 0,60 m para as alturas de 50, 40 e $30 \mathrm{~cm}$, respectivamente, proporcionando assim redução de deriva.

Para as pressões de 200, 300 e $400 \mathrm{kPa}$, observa-se que a porcentagem de volume depositado nas canaletas da mesa de teste foi semelhante, com deposição descontínua, decrescendo do centro para as extremidades (Figura 1). Bicos com esse padrão de deposição são recomendados, segundo Matuo et al. (2001), para trabalhar em barras, havendo sobreposição entre eles. Entretanto, apesar do padrão de deposição semelhante, a vazão cresceu com o aumento da pressão (Tabela 2). Bauer \& Raetano (2004) também verificaram perfis de distribuição de pulverização semelhantes em pontas XR 8004 e TP 8004 submetidas às pressões de 200 e $300 \mathrm{kPa}$.

A ponta de pulverização TT 11002 apresentou uniformidade de distribuição com CV inferior a 7\%, operando na pressão de $100 \mathrm{kPa}$ com bicos espaçados de $50 \mathrm{~cm}$ na altura de barra de $40 \mathrm{~cm}$ e com bicos espaçados de 40, 50 e $100 \mathrm{~cm}$ na altura de barra de $50 \mathrm{~cm}$ (Tabela 1). No entanto, deve-se evitar a utilização do espaçamento de $100 \mathrm{~cm}$, a $100 \mathrm{kPa}$, pois, em razão do perfil de distribuição contínuo, pequenas alterações na altura da barra durante a pulverização implicam redução acentuada na uniformidade de distribuição, conforme ilustrado na Figura 2.
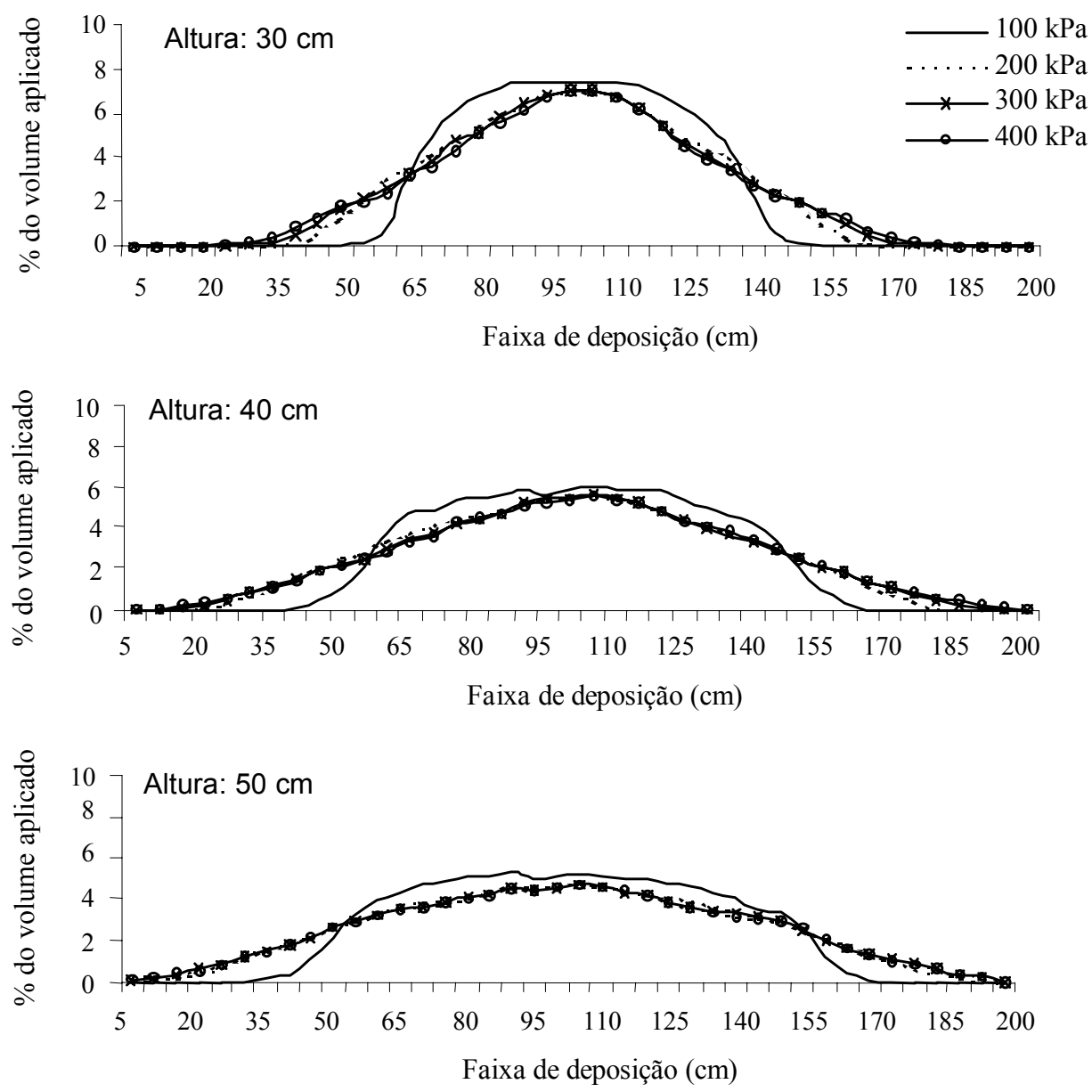

Figura 1 - Perfil de distribuição de pontas TT 11002, operando nas pressões de 100, 200, 300 e 400 kPa, a 30,40 e 50 cm de altura. 
Tabela 1 - Uniformidade de distribuição da ponta de pulverização TT 11002 nas pressões de 100, 200, 300 e 400 kPa, a 50 , 40 e $30 \mathrm{~cm}$ de altura de altura de barra, espaçados de 40,50,80, 100, 120 e $150 \mathrm{~cm}$ na barra de pulverização

\begin{tabular}{|c|c|c|c|c|c|c|c|c|c|}
\hline \multirow{3}{*}{$\begin{array}{c}\text { Pressão } \\
(\mathrm{kPa})\end{array}$} & \multirow{3}{*}{$\begin{array}{c}\text { Espaçamento } \\
\text { entre bicos } \\
(\mathrm{cm})\end{array}$} & \multirow{2}{*}{\multicolumn{3}{|c|}{$\begin{array}{c}\text { Coeficiente de variação }(\%) \\
\text { Altura de barra }(\mathrm{cm})\end{array}$}} & \multirow{3}{*}{$\begin{array}{l}\text { Pressão } \\
\mathrm{kPa}\end{array}$} & \multirow{3}{*}{$\begin{array}{l}\text { Espaçamento } \\
\text { entre bicos } \\
(\mathrm{cm})\end{array}$} & \multirow{2}{*}{\multicolumn{3}{|c|}{$\begin{array}{c}\text { Coeficiente de variação (\%) } \\
\text { Altura de barra }(\mathrm{cm})\end{array}$}} \\
\hline & & & & & & & & & \\
\hline & & 50 & 40 & 30 & & & 50 & 40 & 30 \\
\hline 100 & 40 & 5,19 & 7,30 & 8,81 & 300 & 40 & 2,68 & 0,87 & 2,05 \\
\hline 100 & 50 & 5,03 & 6,37 & 18,78 & 300 & 50 & 1,75 & 2,25 & 5,36 \\
\hline 100 & 80 & 18,45 & 12,04 & 22,73 & 300 & 80 & 3,80 & 1,69 & 8,68 \\
\hline 100 & 100 & 6,49 & 17,85 & 51,83 & 300 & 100 & 5,20 & 6,04 & 26,74 \\
\hline 100 & 120 & 26,54 & 45,49 & 72,65 & 300 & 120 & 5,98 & 19,86 & 45,88 \\
\hline 100 & 150 & 58,97 & 74,79 & 104,01 & 300 & 150 & 24,76 & 40,05 & 62,91 \\
\hline 200 & 40 & 1,23 & 1,82 & 5,21 & 400 & 40 & 2,70 & 1,49 & 1,97 \\
\hline 200 & 50 & 1,25 & 4,01 & 2,05 & 400 & 50 & 2,73 & 1,25 & 5,55 \\
\hline 200 & 80 & 5,62 & 5,19 & 7,42 & 400 & 80 & 3,50 & 1,80 & 10,09 \\
\hline 200 & 100 & 6,89 & 5,50 & 27,80 & 400 & 100 & 5,02 & 5,82 & 25,11 \\
\hline 200 & 120 & 6,98 & 20,67 & 50,61 & 400 & 120 & 6,56 & 19,07 & 43,03 \\
\hline 200 & 150 & 31,71 & 50,45 & 83,21 & 400 & 150 & 36,09 & 41,61 & 53,25 \\
\hline
\end{tabular}

Nas pressões de 200, 300 e $400 \mathrm{kPa}$, uniformidade de distribuição com CV menor que $7 \%$ foi verificada para espaçamentos de até 50,100 e $120 \mathrm{~cm}$, nas alturas de 30,40 e $50 \mathrm{~cm}$, respectivamente (Tabela 1), conforme ilustrado na Figura 3.

Com a variação da pressão entre 200 e $400 \mathrm{kPa}, \mathrm{o} \mathrm{CV}$ ao longo da barra foi semelhante para as diferentes alturas e espaçamentos entre bicos, embora o aumento da pressão implique aumento do volume de calda e maior número de gotas com diâmetros menores, melhorando o grau de cobertura do alvo. Entretanto, segundo Matuo et al. (2001), a utilização de gotas menores implica certas limitações, entre as quais a evaporação e a deriva são as principais. Assim, na escolha da pressão de trabalho, nesse intervalo, deve-se levar em conta o grau de cobertura do alvo que se pretende alcançar e os riscos inerentes à deriva.

Com a boa uniformidade de distribuição obtida para os espaçamentos entre bicos de 80,100 e $120 \mathrm{~cm}$, verifica-se na Tabela 2 a possibilidade de redução do volume de aplicação através do aumento do espaçamento entre bicos, onde estão apresentados os valores obtidos a partir das vazões médias verificadas para as pressões de 100, 200, 300 e $400 \mathrm{kPa}$, para os espaçamentos entre bicos que apresentaram $\mathrm{CV}$ inferior a $7 \%$, nas velocidades simuladas de 4 e $6 \mathrm{~km} \mathrm{~h}^{-1}$. Esses resultados estão de acordo com Ferreira et al. (2003), que recomendam a utilização de pontas TT espaçadas de $100 \mathrm{~cm}$ como alternativa para redução de volume de calda em aplicação de herbicidas, resultando em menor transporte de água para o campo e redução das paradas para reabastecimento do pulverizador, obtendo-se, com isso, diminuição do custo da aplicação e aumento da capacidade operacional (Matthews, 1979; Marochi, 1993; Lima \& Machado Neto, 2001), o que pode, em muitos casos, evitar a compra de tratores e pulverizadores (Marochi, 1993).

Em trabalhos similares com outras pontas de pulverização de menor ângulo de abertura, Perecin et al. (1994) e Bauer \& Raetano (2004) alertam para a conveniência de utilizar espaçamentos entre bicos inferiores a $50 \mathrm{~cm}$ a fim de conseguir os niveis de CV desejados. Entretanto, para a ponta TT 11002, maiores espaçamentos entre bicos podem ser utilizados graças ao maior ângulo de abertura, que produz uma faixa de deposição mais larga, atingindo aproximadamente dois metros nas pressões de 200, 300 e $400 \mathrm{kPa}$ a $50 \mathrm{~cm}$ de altura (Figura 1), aumentando a sobreposição entre bicos (Figura 3).

Planta Daninha, Viçosa-MG, v. 23, n. 1, p. 161-167, 2005 

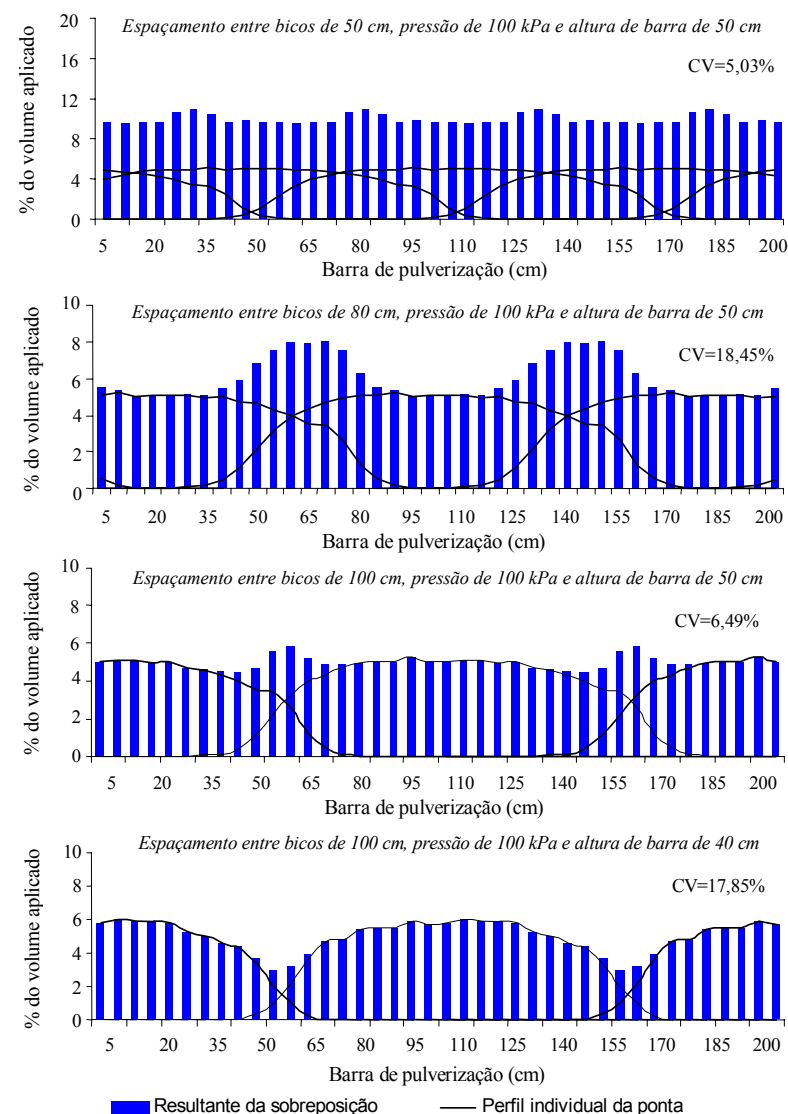

Figura 2 - Distribuição do volume pulverizado ao longo da barra de pulverização, com pontas TT11002 em diferentes espaçamentos e alturas de barra, na pressão de $100 \mathrm{kPa}$.
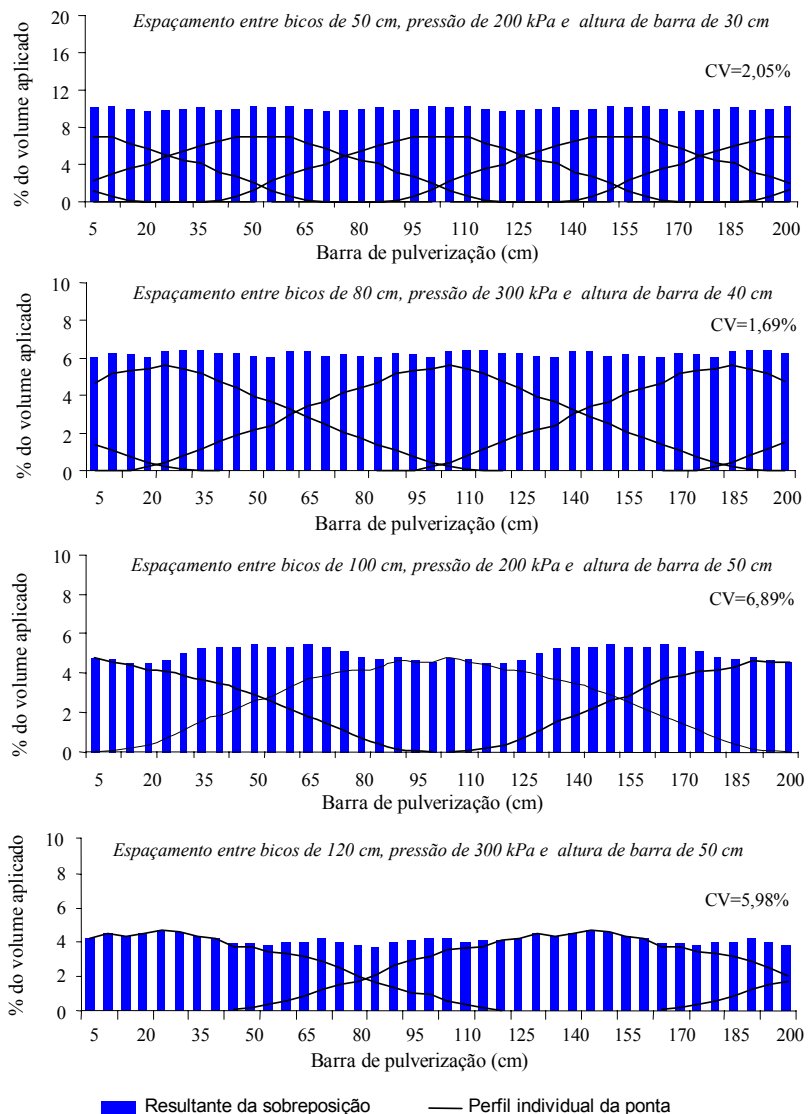

Figura 3 - Distribuição do volume pulverizado ao longo da barra de pulverização, com pontas TT11002 em diferentes pressões de trabalho, alturas de barra e espaçamentos entre bicos.

Tabela 2 - Volume de calda, em L ha ${ }^{-1}$, obtido a partir das vazões médias verificadas para as pressões de 100, 200 , 300 e $400 \mathrm{kPa}$, para os espaçamentos de 40, 50, 80, 100 e $120 \mathrm{~cm}$, nas velocidades de 4 e $6 \mathrm{~km} \mathrm{~h}^{-1}$

\begin{tabular}{|c|c|c|c|c|c|c|c|c|c|c|c|}
\hline \multirow{3}{*}{$\begin{array}{c}\text { Pressão } \\
(\mathrm{kPa})\end{array}$} & \multirow{3}{*}{$\begin{array}{c}\text { Vazão } \\
\left(\mathrm{L} \mathrm{min}^{-1}\right)\end{array}$} & \multicolumn{10}{|c|}{ Volume de calda em $\left(\mathrm{L} \mathrm{ha}^{-1}\right)$} \\
\hline & & \multicolumn{2}{|c|}{$40 \mathrm{~cm}$} & \multicolumn{2}{|c|}{$50 \mathrm{~cm}$} & \multicolumn{2}{|c|}{$80 \mathrm{~cm}$} & \multicolumn{2}{|c|}{$100 \mathrm{~cm}$} & \multicolumn{2}{|c|}{$120 \mathrm{~cm}$} \\
\hline & & $4 \mathrm{~km} \mathrm{~h}^{-1}$ & $6 \mathrm{~km} \mathrm{~h}^{-1}$ & $4 \mathrm{~km} \mathrm{~h}^{-1}$ & $6 \mathrm{~km} \mathrm{~h}^{-1}$ & $4 \mathrm{~km} \mathrm{~h}^{-1}$ & $6 \mathrm{~km} \mathrm{~h}^{-1}$ & $4 \mathrm{~km} \mathrm{~h}^{-1}$ & $6 \mathrm{~km} \mathrm{~h}^{-1}$ & $4 \mathrm{~km} \mathrm{~h}^{-1}$ & $6 \mathrm{~km} \mathrm{~h}^{-1}$ \\
\hline 100 & 0,424 & 159 & 106 & 127 & 85 & - & - & - & - & - & - \\
\hline 200 & 0,636 & 239 & 159 & 191 & 127 & 119 & 80 & 95 & 64 & 80 & 53 \\
\hline 300 & 0,785 & 294 & 196 & 236 & 157 & 147 & 98 & 118 & 79 & 98 & 65 \\
\hline 400 & 0,920 & 345 & 230 & 276 & 184 & 173 & 115 & 138 & 92 & 115 & 77 \\
\hline
\end{tabular}

A ponta TT 11002 é uma alternativa para redução de volume de calda através do aumento do espaçamento entre bicos, mantendo boa uniformidade de distribuição com maior capacidade operacional do equipamento de aplicação. Permite redução de deriva, uma vez que pode trabalhar mais próxima do alvo e com pressões menores, podendo também ser usada na aplicação de herbicidas em faixa, na pressão de $100 \mathrm{kPa}$. 


\section{LITERATURA CITADA}

BAUER, F. C.; RAETANO, C. G. Distribuição volumétrica de calda produzida pelas pontas de pulverização XR, TP e TJ sob diferentes condições operacionais. Planta Daninha, v. 22 , n. 2, p. 275-284, 2004.

CARLSON, K. L.; BURNSIDE, O. C. Comparative phytotoxicity of glyphosate, SC-0224, SC-0545, and HOE0661. Weed Sci., v. 32, p. 841-844, 1984.

CORDEIRO, A. M. C. Como a tecnologia de aplicação de produtos fitossanitários pode contribuir para o controle de pragas, doenças e plantas daninhas. In: ZAMBOLIM, L. Manejo integrado: fitossanidade, cultivo protegido, pivô central e plantio direto. Viçosa-MG: UFV, 2001. p. $683-721$.

FERREIRA, L. R.; FREITAS, F. C. L.; FREITAS, L. H. L. Técnicas de aplicação de herbicidas em plantio direto. Brasília: SENAR, 2003. 78 p.

FURLANETTI, A. C.; MATUO, T.; BARBOSA, J. C. Uniformidade de deposição da calda de pulverização de herbicidas em barra lateral protegida com diferentes combinações de pontas de pulverização. Planta Daninha, v. 19, n. 3, p. 445-455, 2001.

LIMA, P. R. F.; MACHADO NETO, J. G. Otimização da aplicação de fluazifop-p-butil em pós-emergência na cultura da soja (Glycine max). Planta Daninha, v. 19, n. 1, p. 85-95, 2001.
MAROCHI, A. I. Tecnologia de aplicação de defensivos agrícolas. In: SIMPÓSIO INTERNACIONAL SOBRE SEMEADURA DIRETA EM SISTEMAS SUSTENTÁVEIS, 1993, Castro-PR. Anais... Castro-PR: Fundação ABC, 1993. p. 208-227.

McWHORTER, C. G.; HENKS, J. E. Effect of spray volume and pressure on postemergence johnsongrass (Sorgum halepense) control. Weed Technol., v. 7, p. $304-310,1993$.

MATUO, T.; PIO, L. C; RAMOS, H. H.; FERREIRA, L. R. Tecnologia de aplicação e equipamentos. In: ABEAS Curso de proteção de plantas. Módulo 2. Brasília, DF: ABEAS; Viçosa, MG: UFV, 2001. 85 p.

MATTHEWS, G. A. Droplets. In: Pesticide application methods. London: 1979. p. 57-74.

PERECIN, D. et al. Padrões e distribuição obtidos com bicos Twinjet em função da altura e do espaçamento entre bicos. Eng., Agric., v. 14, p. 19-30, 1994.

SOUZA, R. O.; DORNELES, S. H. B. Influência do volume de calda na mistura de herbicidas pós-emergente na soja (Glicine $\max (\mathrm{L}$.$) Merril). In: CONGRESSO BRASILEIRO$ DE HERBICIDAS E PLANTAS DANINHAS, 20., 1995, Florianópolis. Palestras... Florianópolis: SBCPD, 1995. p. 41.

SPRAYING SYSTEMS CO. Produtos de pulverização para agricultura - Catálogo 46M-BR/P. 1999. 